\title{
THE REDUCTION(?) OF THERMODYNAMICS TO STATISTICAL MECHANICS
}

It is now very well understood in philosophy that the relationships that related but distinct theories bear to one another is a matter of enormous complexity. Whatever the relationship is between a theory and another that somehow or other "grounds" it, the terms "reduction' and 'supervenience' and 'emergence' are all so broad in their sweep that to say that one theory reduces to another, is supervenient on another or emerges from another is never more than to begin to tell the truly interesting story: Exactly how is the one theory related the other. Often much of the most interesting philosophical insight resides in the details of the specific cases rather than in categorizing some particular inter-theoretic relation into one of the three broad categories mentioned.

Much of the realization that inter-theoretic relations are as complex as they are has come from the exploration of the relation of the special sciences to one another and to physics. Exactly how a conceptual scheme appropriate to deal with social communities is to relate to individualistic psychology, and, notoriously, how a psychology replete with talk of states endowed with cognitive content and with states endowed with perceptual content is to related to a theoretical account of the psychologically endowed as brain possessing biological organisms embedded in their complex of relations with other such organisms and with the physical world, has proven about as intractable problem area as any in philosophy. Similarly the relationship biology bears to the underlying chemistry and physics of living things has shown itself ever more complex the deeper our understanding goes.

Philosophical Studies 95: 187-202, 1999.

(C) 1999 Kluwer Academic Publishers. Printed in the Netherlands. 
But even within physics itself, the more we probe into intertheoretical relations the harder we find it to characterize them in all but the most general terms if we want analyses of these relations that will hold across numerous important cases. Instead we find that the individual cases each present a complex of subtle details and that it is in these details that much of the most interesting results lie in trying to understand just how the one theory is related to the other.

This is true in both of two kinds of important cases. First there is the situation where we have a kind of "replacement reduction." In these cases we have a newer theory supplanting an older theory, but where the older theory remains "true enough" in appropriate contexts so that we don't want to speak of its being utterly refuted (as are the theories, say, of crystalline spheres in astronomy or caloric in thermal physics). Rather we think of the older theory as being "reduced" to the newer alternative in some sense. Second there are the cases where we think of the newer theory as, if anything, providing even greater support for the older one, if, perhaps the older one in a modified form, rather than showing us the error of its ways.

In the first group of cases there is enormous variety. The relationship of pre-relativistic theories of space time and motion to the spacetime theories of the special and the general theory of relativity has been studied in great detail by physicists and philosophers. Here, at least, some general patterns of the kind we would naively expect antecedent to looking at the details can be found. Pre-relativistic theory and the spacetime of special relativity can, for example, be neatly related to one another by letting a parameter, the velocity of light, go to infinity.

But other fundamental cases are much more problematic. Just exactly how does pre-quantum dynamics relate to the quantum theory? In a narrow class of special models within the theories there is, once again, a neat "parameter limit" connection between the descriptions of the case given by the two theories. But in other cases getting straight just how the theories are connected requires great subtlety indeed. The relation of a "wave" theory to one without the characteristic wave phenomena, or of a theory framed in terms of observables characterized by functions on phase points to one where they are characterized by operators on a vector space, is 
still an area of intense scientific exploration. There is, for example, active research dedicated to finding out just how to characterize at the quantum level those systems characterized classically by chaotic dynamics. All of which is not to recommend, of course, the counsel of despair called "incommensurability."

But there are deep problems with the other kinds of cases as well, the cases where we think of the "reducing" theory not as supplanting but as grounding the theory "reduced." I'll spend the rest of this paper dealing with one such, particularly problematic, case.

Thermodynamics is the general theory of energy, whether in the form of overt mechanical work or in the form of transferable heat, and its transformations and inter-relations with the other macroscopic descriptive parameters of matter and radiation. It is a very odd theory indeed. It doesn't fit either into the class of "constitutive" theories describing the various entities in the world and their structure. Nor does it fit into the scheme of the hierarchies of ever deeper dynamical theories that describe the causal order among events at deeper and deeper levels. Yet its idiosyncratic general principles, especially the Second Law of Thermodynamics with its description on an "irreversible," time-symmetric world, is applicable across the entire range of physical phenomena.

Statistical mechanics is equally peculiar. Once again it has universal applicability across all of the domains of physics. Yet, once again, it seems to have no place in the usual hierarchy of constitutive or dynamical theories. It presupposes both of these in essential ways. Two essential ingredients assumed by the theory are: (1) The reductive account of macroscopic matter that views it as being constituted of microscopic components that are related to one another by various forces; and (2) An assumed theory of dynamics that expresses the laws governing the time evolution of systems composed of such components interacting in such ways. This latter component of the theory was, originally, taken to be classical mechanics and is now taken to be the appropriate quantum dynamics for the microscopic components of the system. 
Whether or not thermodynamics reduces to statistical mechanics, then, the very possibility of statistical mechanics rests upon the presupposed identification of macroscopic matter with an assemblage of microscopic parts, an identification essential to other reductive aspects of physics.

What is most peculiar about statistical mechanics, though, is its assumption of various probabilistic posits that, under most interpretations of the theory, fail to have their complete grounding in either the theory of the constitution of matter or in the underlying dynamics. But the autonomy of such probabilistic posits, posits of quite different kinds in different contexts of the theory, is itself a matter of great controversy. It is, in fact, the status of these probabilistic assumptions, central to the theory and possibly not importable into it from other physical theories, that is the most problematic element when one asks whether we ought to claim a reductive relationship between thermodynamics and statistical mechanics. I'll return to this in the later sections of this paper.

If we want to speak of one theory as in any sense reducible to another, and not just supervenient on the other, then we need to understand the connections between the concepts of the reduced and those of the reducing theories. There are, indeed, such inter-theoretic conceptual relations between thermodynamics and statistical mechanics. But they have a complexity and variety that cannot be summarized in any simple form.

Some of the concepts of thermodynamics, such as volume, pressure and energy are imported into that theory from the outside from our general spatial language or from the language of dynamics. Other thermodynamic concepts such as temperature and entropy are novel to the theory. How do they acquire their meanings in the theory? Their evolution as the theory develops shows a familiar pattern. Some of them arise first as modes of description of immediate experience (temperatures as hot or cold, for example). Then that sense is removed from them and their meaning is fixed by the measuring instruments used to determine their quantitative values in a familiar operationalist way (temperature as that 
which is measured by a gas thermometer, for example). Then as the theory becomes formalized their meanings are fixed by their role in the basic assumptions of the theory (the place of temperature, for example, in the Zeroth and Second Laws of Thermodynamics). In the case of entropy (and many other related concepts), earlier stages of meaning accrual don't exist at all. The concept first appears in its role as theoretical place-holder. Not surprisingly for the later uses of concepts and for concepts that never had any meaning other than their role in the theory, an analysis of their meaning something akin to functionalist accounts of mental concepts is appealing.

For some of the thermodynamic concepts, their association to concepts employed in statistical mechanics is, at least in part, fixed by the fact that both theories exist in the context of surrounding general kinematic and dynamical theory from which common terms were imported into both theories. When we speak of the volume of a gas in thermodynamics and look for the appropriate concept to associate with that in statistical mechanics, we find in the new theory that the spatial discourse has already been embedded and that an inevitable statistical mechanical notion of volume exists to be associated with the thermodynamic concept.

But even here we must be careful. For a concept such as volume can function at the statistical mechanical level in much subtler ways that it does in thermodynamics. In statistical mechanics we deal with imagined collections of systems, or, more directly, with probability distributions over microscopic states compatible with some constraint on systems such as fixed volumes or energies. Volume could function as a constraint on such a collection of systems. Or it might function, instead, as some quantity allowed to vary from collection member to collection member, where the collection is specified by constraints on its members not including fixed volume. If the latter is the case, volume will be subject to all the probabilistic aspects of statistical mechanics. That is observations of volume will be connected to such things as mean or most probable values of volume and fluctuations of volume as a measurable quantity will be tolerated.

Here we encounter one of the fundamental features making the relation of thermodynamics to statistical mechanics irremediably complex. One of the theories is framed in fundamentally probabil- 
istic terms and the other, at least in its standard form, is not. So even when we are pointed to an immediate association of concepts at the two theoretical levels, that association can in no way be mediated by some simple notion of alleged synonymy of terms or even of property identification.

When we deal with the concepts of thermodynamics imported from the more general physics of motion, that is to say kinematic and dynamical concepts such as energy or pressure, the situation is quite like that when dealing with volume. The statistical mechanical theory imports concepts from general dynamics just as thermodynamics does. Therefore which concept of thermodynamics is to be associated with which concept of thermodynamics is once again fixed to a degree by the physics external to both theories.

But, once again, no simple notion of synonymy or property identification will tell the whole story. As an example consider the transfer of energy into or out of a system. In thermodynamics this can take two forms, either that of overt mechanical (or other) work done on or by the system, or as the transfer of heat. But the distinction between these two kinds of energy transfer, built into thermodynamics just as the posit that energy as a whole is conserved with heat transfer being what is left over when overt work is subtracted from total energy transfer, becomes much more deeply understood from the statistical mechanical point of view. Here work becomes the "controllable" transfer of energy of the system as a collective and heat the transfer of that part of the energy distributed over the microscopic constituents of a system in a "random" or "uncontrollable" way. Making clear sense of this is no easy task, and one that, once again, requires probing into the way in which probabilistic considerations and dealing with probability distributions over quantities of systems rather than with those quantities themselves is central to statistical mechanics.

The problem of explicating the relationship between thermodynamics as a non-probabilistic theory with probabilistic statistical mechanics makes it one of those "special difficulties" in accounting for inter-theoretic relationships. In this way, but only in this way, it resembles the special problem encountered in trying to make sense of the relationship between classical mechanics, which is devoid 
of wavelike aspects and the wavelike quantum mechanics to which classical mechanics is supposed, in some sense or other, to reduce.

It might be added here that the central probabilistic aspect of statistical mechanics has led, in a familiar way, to the reconstruction of thermodynamics itself in a probabilistic manner. A theory of statistical thermodynamics has been constructed that uses a small number of elementary posits to introduce into thermodynamics itself a sufficiently probabilistic aspect to make the relationship between this newly reconstructed theory and statistical mechanics more direct than that between traditional thermodynamics and statistical mechanics. Fluctuational phenomena, for example, now become overt at the purely thermodynamic level. Needless to say, even when so reconstructed, the relationship between thermodynamics and statistical mechanics remains fraught with special problems.

When we move from the concepts, such as volume, energy and force, imported into both theories from external physics to such purely thermodynamic concepts as temperature and entropy, the inter-theoretic conceptual relations take on a different aspect. In these cases we have much less guidance from the outside about which concepts available at the statistical mechanical level to associate with some purely thermodynamic concept at the thermodynamic level. Among other things this sometimes leaves us with a situation where a multiplicity of concepts that reside at the statistical mechanical level, and that are certainly not equivalent at the level, can each be argued to be appropriately associated with a single thermodynamic concept. Here the condition of appropriateness may itself be context dependent in several ways.

The concept of temperature in thermodynamics has its meaning fixed initially be the specification of appropriate thermometers, temperature measuring devices, in the familiar operationalist way. Later, and more importantly, the concept has its primary role fixed by its functional place in the network of thermodynamic laws. Once accept the notion of an equilibrium state for a system and then accept a principle of transitivity for the relationship of one system being in equilibrium with another, then a notion of empirical temperature as 
marker of equilibrium states follows. Add the Second Law and a notion of absolute temperature can be derived as Clausius showed relying on the work of Carnot.

When we move to statistical mechanics we see many subtleties in trying to identify which concepts available in that theory ought to be the ones associated, one way or another, with temperature in thermodynamics.

One such subtlety will come as no surprise to philosophers. That is multiple realizability. Systems of wildly divergent physical kinds can all have equilibrium states and, when allowed to transfer energy to one another, be in equilibrium with respect to each other. The moving molecules in a blob of matter, for example, can form a system in equilibrium with electromagnetic radiation. So we are required to think of these systems as sharing common temperatures. So a given temperature can be realized in radically different physical ways. There is a clear resemblance between this multiple realization in a variety of physical ways of property whose specification is functionally given by the role played by a concept in a network of laws and the claim that mental states embodying cognitive content could achieve their realizations in a variety of physically unlike organisms. And, just as in the case of the relationship between the mental and the physical, this multiple realizability makes a too naïve claim of the identity of the thermodynamic property with some single variant of one of its realizing physical properties misleading. We must move to some subtler notion of context-relative identifications or determinable-determinate relations. So much for "temperature just is mean kinetic energy of molecules."

The complexities introduced by the probabilistic nature of statistical mechanics, though, have no counterpart in the mind-body case. And they present a deeper challenge to understanding how temperature in thermodynamics is to be related to temperature in statistical mechanics than do the issues of multiple realizability.

One role temperature can play in statistical mechanics is as a measure of some property instantiated in individual systems. In that guise it is an ordinary sort of physical property. And it is subject to the probabilistic, fluctuational behavior of most properties of individual systems. The regularities in which it appears will now be statistical regularities, and the association of these new laws with the 
traditional laws of thermodynamics will be less immediate than any simple identification of the latter with the former. Heat, for example, most certainly can, and does, flow from a colder to a warmer body in an isolated system in the new probabilistic framework.

But the more important role for temperature in statistical mechanics is as a way of characterizing ensembles, that is as a parameter appearing in some appropriate probability distribution over the microscopic states of individual systems. Here the relationship between a specified temperature and an individual system is quite indirect. One can think of such an individual system as having a certain temperature, that is as being appropriately dealt with as being a member of a collection of systems specified by a probability distribution with that temperature as parameter, even if the individual system has a temperature in the individual system sense wildly divergent from the ensemble temperature. But with temperature, and other thermodynamic quantities, thought of in this new way, one may find in statistical mechanics new laws that match the older laws of thermodynamics without any probabilistic qualification. The "probabilism" has already been built in to the nature of the concepts as ensemble parameters.

The connections between the thermodynamic concept of entropy and those concepts of statistical mechanics associated with it are of such complexity, and such importance, as to deserve special attention on their own.

In thermodynamics the concept of entropy is defined solely by its function in the theory. We have no direct phenomenal sense of entropy, nor are there devices that serve as direct entropy measurers. Crudely entropy measures the degree to which matter or energy is spread out in a system. Gas spread throughout a box has a higher entropy than gas confined to a portion of the box. A bar warm all over has higher entropy than a bar with the same internal energy concentrated in a hot end with the remainder of the bar cold. The non-decrease of entropy in any process in an isolated system is, of course, the most fundamentally special thermodynamic aspect of the world, its asymmetry in time. 
What functions as the correlate of entropy in statistical mechanics? The only correct answer is: "Lots of different things." For different purposes, in different contexts, with different presuppositions, many quite distinct concepts can all be identified as the statistical mechanical entropy.

To begin with there is the distinction between entropy as a property of a system and entropy as characterizing an ensemble, a probability distribution, into which systems of a given kind are assembled. This parallels the similar distinctions noted above and once more gives rise to the distinction between properties that are now thought of as fluctuating in an individual system and properties that have the fluctuation structure built into their very definition.

The multiplication of entropies doesn't stop there. In formal thermodynamics entropy is a property that can be legitimately assigned only to a system in equilibrium, although extensions to non-equilibrium systems can sometimes be made. In statistical mechanics one would like to assign entropy to systems not in equilibrium and to then derive an appropriate increase of entropy for an isolated system undergoing an irreversible change. But when one applies to the description of non-equilibrium systems the most natural property available to describe ensembles in statistical mechanics that one would associate with the thermodynamic entropy, it turns out that it is easily provable that the quantity in question must remain unchanged in any dynamic process.

Following an idea in Gibbs and implicit perhaps even in Boltzmann, a new, "coarse grained" entropy is invented. When a system is in equilibrium this coarse grained entropy will coincide with the original "fine grained" entropy. But when constraints are changed and a probability distribution changes under the dynamics governing the individual systems in an ensemble, the coarse grained entropy can nicely increase even if the fine grained remains provable constant.

Dealing with this explosion of concepts is no easy task. First one has to show that the coarse grained entropy will really do what one wants it to do, that is to change in a way that will correctly represent the irreversible dynamical change characteristic of thermodynamic systems. Making that work requires the subtlest considerations imaginable of both the underlying dynamics and of 
the initial probability attributed to the initial states of systems. On top of that one needs to show that there are ways of construing this new concept so that it is reasonable to associate its changes conceptually with the kind of changes observationally available to us when macroscopic systems are observed. Neither the dynamics nor the conceptual issues are settled matters, and the appropriate role for such a coarse grained notion is still controversial in the foundations of statistical mechanics.

But, the conceptual complexities don't even end there. There exist systems that can be forced to show apparent "antithermodynamic" behavior. Despite the general irreversibility of systems, fluctuation to the side, these unusual systems can show behavior that seems to go the wrong way in time in terms of the usual transition from order to disorder. How should they be described at the statistical mechanical level? One choice is to stick with standard definitions of entropy. One then simply denies the universal validity of the Second Law of Thermodynamics, even construed as a probabilistic regularity. But an alternative exists in which one reconstrues the definition of entropy in the theory in just such a way that the overall evolution of the system can be considered one in which entropy never does decrease in the future time direction.

It would be deeply misleading to ask which of the many entropies of statistical mechanics is the right one, or even to ask which one corresponds or can be identified with the familiar entropy of thermodynamics. Once the newer theory is available to us we realize that the world is much more complicated that we originally thought, and that a richer conceptual scheme is needed to deal with all its aspects.

Despite all of this let me state my strong disagreement with any claim to the effect that such complex relations between the theories at the two levels can be dealt any kind of justice by taking about incommensurability. It is not the absence of meaning relations between the theoretical terms of the two theories we are taking about. To the contrary, it is the just the richness and complexity of such meaning relations that require our attention if we are to make any kind of reasonable appraisal of the vague claims that the one 
theory is or is not reducible to the other or to the effect that there is some kind of emergence going on.

In terms of the relation of concepts at the supervenient level to those at the grounding level, then, the relationship of thermodynamics to statistical mechanics is neither one of a simple concept-concept association nor that of there being concepts at the supervenient level that have no natural correlates at the grounding level. Instead there is a network of complex relationships between the concepts of the older theory and a proliferation of novel concepts in the newer.

A series of questions, perhaps more profound than those dealing with conceptual relations between the theories, centers around the issue of whether it is correct, and if so in what sense it is correct, to think of thermodynamics as being supervenient in any sense on statistical mechanics. It isn't clear how to answer these questions at the present time. For one thing, there really isn't any general agreement about just what the theory of statistical mechanics is. And the disagreements here are such that they entail serious disagreement about the extent to which the thermodynamic regularities, including irreversibility, can be thought of as being explained in any way by resort to statistical mechanics.

One version of statistical mechanics seeks its probabilistic nature and its irreversibility in the irreducibly probabilistic and irreversible nature of some fundamental dynamical law of nature. Once upon a time aspects of radiation were suggested as doing the trick. At other times quantum mechanics was suggested as supplying the needed asymmetrically, chancy aspects of the world. The most plausible candidate now is a highly speculative theory introduced originally to account for the mysterious process of measurement in quantum mechanics. This Ghirardi-Rimini-Weber theory introduces a lawlike, stochastic process that is time reversal non-invariant and that disturbs what would otherwise be the deterministic evolution of the wave-function described by quantum mechanics. It is this disturbance that can make macroscopic systems really collapse into eigenstates whereas microscopic systems remain happily in superpositions. Recently David Albert has suggested that the GRW 
process - if it is real - might account for some of the stochastic irreversible aspects of statistical mechanics as well.

If this were so, and believing that it is so requires both believing that the GRW theory is true and that it can do the job allotted to it by Albert, we would then have a pretty clear case for the supervenience of thermodynamics on an underlying fundamental dynamical theory. Since that underlying theory would be irreducibly tychistic, there would be some interesting variation here on the usual supervenience theme, but nothing, I think, that would lead us to believe that some real kind of lack of supervenience of a plausible sort could be alleged.

Another interpretation of statistical mechanics might serve to ground an uncomplicated supervenience claim if it were correct. There have been some, Prigogine for example, who would argue that our positing of exact microscopic dynamical states for systems is a false idealization. From this perspective there are no such exact dynamical states. Instead each individual system has as its dynamical state at a time something that can be correctly characterized itself by a probability distribution over a range of ordinary dynamical microstates.

What this interpretation amounts to is far from clear, since we are never told exactly how to think of the irreducible "spread" microstates of individual systems. In addition, there are a number of reasons for thinking the interpretation untenable. In a number of contexts the need for thinking of the exact microstates of the traditional theory as genuine seems strongly supported. But were such an interpretation both intelligible and true, it seems likely that some claim of immediate supervenience would hold between the statistical mechanical theory so interpreted and the theory of macroscopic thermodynamics.

But what should we say if we adopt the most common, orthodox interpretation of statistical mechanics? Here one takes it that one cannot find the source of the fundamental probabilities of statistical mechanics in any tychistic nature of the underlying dynamical laws. And systems are viewed as having the exact microstates posited by the underlying dynamics. Where then is the source of the probabilistic nature of the theory? 
It is most often taken to be in the distribution over initial conditions of systems prepared in a non-equilibrium state. Although some quasi-probabilistic consequences can be derived from the dynamics alone, both in equilibrium and non-equilibrium theory, in the form of ergodic theorems or theorems about the "mixing" nature of a system, the general consensus is that one cannot get the full non-equilibrium theory without making some basic posit about frequencies or proportions with which initial conditions are realized in the world when the system is prepared in a non-equilibrium condition. Only with such a posit can we get the appropriate probabilistic evolution toward equilibrium with its characteristic relaxation time and characteristic flow behavior.

But why are initial conditions distributed in the way they are? It is here that there seems to many to be an ineliminable posit in the theory that has its origin outside of anything we can say about how the systems are constituted, how they are prepared, or how the dynamical laws govern their evolution. It is this, I think that is the primary reason that so many practitioners in the field are wary of claiming any genuine kind of reducibility in the underpinning of thermodynamics by statistical mechanics. The implicit thought seems to be that the very nature of statistical mechanics requires that we make in it a basic assumption whose independence of the remainder of physics at that reductive level almost makes it looks as though we are presupposing a kind of thermodynamic truth rather than deriving it.

Unless we can discharge such a posit about distribution of initial conditions, many seem to think, it is misleading of us to think that the fundamental thermodynamic truths have really been explained. Rather, they have been assumed at the lower level theory in a novel form in the posit about proportions among initial conditions that is super-added to all the remaining physics dealing with how macroscopic things are made up out to their microscopic parts and how these microscopic parts behave in a lawlike way.

There is more, then, to the suspicions about the irreducibility of thermodynamics to statistical mechanics than the kind of objection to irreducibility that comes about from the fact that the concepts of the special science have no natural correlate in the concepts framing the more universal theory. In the thermodynamics-statistical 
mechanics case there are indeed important questions about typetype relations. But there are natural associations of concepts at the thermodynamic level with those of the statistical mechanical level complex as they may be. It isn't at all like the relation of the "theory of furniture" to the theory of atoms.

It is the curious need for a posit at the lower level that seems to come from nowhere as far as the lawlike structure of that lower level is concerned that makes a kind of emergentist claim for the thermodynamic nature of the world appealing to so many. Incidentally all of this occurs before one ever gets into the most famous question of all about the probabilistic distribution of initial conditions, that is, its time asymmetry. Even before one gets to that notorious issue the problem of the source of natural proportions for classes of initial conditions arises.

There is one final matter to note that casts serious doubt upon claims that thermodynamics reduces to statistical mechanics in any simple minded way. In order to carry out the program of nonequilibrium statistical mechanics, we must be able to specify some appropriate initial probability distribution over the microstates of a system. It is in the evolution of such an initial probability distribution that the description of the approach to equilibrium resides. Deriving this evolution is a complicated task. First of all, most standard derivations impose a re-randomization posit (some "Hypothesis of Molecular Chaos") whose very consistency with the laws of dynamics governing the constituents of the system may be in doubt. A rigorous theory would dispense with such a posit, but there is very little in the way of rigorous theory in existence.

But how can we fix on the appropriate initial probability distribution? For most non-equilibrium conditions of systems we have no idea. There are only a limited class of cases where any plausible proposal can be made about such an initial statistical posit. How are these cases discovered? Basically by a combination of experimental experience with the standard thermodynamic ways of characterizing experience. 
If a system is close to equilibrium, a plausible way of generating initial ensembles exists. If the system is far from equilibrium, but is such that small enough pieces of it can be thought of as close to equilibrium for short enough times, then, once again, plausible rules for generating initial probability distributions can be proposed. But we have virtually no guides from the underlying mechanical or statistical mechanical theory as to when such local equilibrium conditions will exist or what they will be like. It is only in already established thermodynamic (and hydrodynamic) description of experimentally tractable systems that we find our guidance about which systems can even get a statistical mechanical description and our guidance as to what that description will be like.

Of course once the new level of statistical mechanical description has been imposed, we gain new insights unattainable at the purely thermodynamic level. In particular, whereas the thermodynamic description relies on the assignment of values of parameters (such as thermal conductivity, viscosity, etc.) obtained by experiment, in the fuller statistical mechanical account these may be derivable by theory from the underlying constitution of the system and the dynamics of the micro-constituents. But the fact that when the statistical mechanical treatments exists, and how they are to be framed when they do, is guided solely by the existing thermodynamics and experiment, and has no derivation from fundamental theory, makes a claim of reducibility of thermodynamics to statistical mechanics dubious in a fundamental way.

\section{POSTSCRIPT}

A more extended treatment of the issues discussed in this paper can be found in chapter 9, 'The Reduction of Thermodynamics to Statistical Mechanics', of the author's Physics and Chance: Philosophical issues in the Foundations of Statistical Mechanics (Cambridge University Press, 1993). The "Further Readings" section at the end of that chapter provides an annotated guide to further reading on the issues dealt with here.

University of Michigan 